\title{
Opportunities multifrequency focusing shock wave therapy in lecheni and intervertebral hernias of the lumbar spine
}

\author{
Summary \\ A new effective method of conservative treatment of hernias of intervertebral discs of \\ the lumbar spine: Multifrequency in general, shock wave therapy.1 Clinical evaluation \\ of the effectiveness of the method. The parameters of the exposure and the steps \\ management of patients with spinal hernias.
}

Keywords: spinal hernia, shock wave therapy, treatment of intervertebral hernia
Volume 3 Issue 5 - 2018

\author{
Ovcharenko Leonid Mikhailovich \\ Chief physician,VR-Clinic, Russia
}

Correspondence: Ovcharenko Leonid Mikhailovich, Chief physician,VR-Clinic, 355037, 50 years of the Komsomol and I6, office 248, Stavropol, Russia, Email r_leonid@mail.ru

Received: November 23, 2017 | Published: October 15, 2018

\section{Introduction}

The problem of intervertebral hernia is quite acute in modern orthopedics and neurology. As Filatov VS notes, hernias of intervertebral discs as the only cause of compression of roots are noted in $38.0 \%$ of observations, $41.5 \%$ of those surveyed have a combination of hernias with other vertebrogenic factors; in $20.5 \%$ of cases, the compression of the roots is due only to non-hernial factors. In general, the causes of compression of the lumbar and sacral roots in the dystrophic pathology of the spine are many. Its causes, in addition to herniated intervertebral discs, are: hypertrophied yellow ligaments, edema, adhesive changes in epidural fiber, varicose dilated epidural veins, osteophytes of vertebral bodies, irregularly formed vertebrae arches, primary stenosis of the spinal canal and various dislocations. ${ }^{2,3}$ Lumbar pain (PB) associated with hernia of the MTD is defined as chronic after 3 months. It is estimated that in $15-20 \%$ of cases, prolonged pain develops, and approximately $2-8 \%$ of patients acquire chronic pain. Chronic back pain is the most common cause of disability in Americans younger than 45 years. Every year, 3-4\% of the US population is temporarily inactive, and $1 \%$ of the workingage population is completely disabled. ${ }^{4}$ Herniated disc hernias are a popular cause of vertebral and radicular pain in practicing doctors and the most common cause of operations in the lumbar spine. But asymptomatic disk hernias on computed tomography (CT) and magnetic resonance imaging (MRI) are found as random findings in a large number of cases. ${ }^{5-8}$ In addition, there is no clear correlation between the degree of prolapse of the disc and clinical symptoms. ${ }^{9}$ Nevertheless, the treatment of hernia of the MTD in most cases of $\mathrm{PB}$ is a top priority. A significant percentage of patients with herniated MTD is operated. Up to $50 \%$ of all surgical interventions in neurosurgical hospitals are performed for protrusion and extrusion of lumbar intervertebral disks. ${ }^{10}$ At the same time, searches for noninvasive methods of treatment of intervertebral hernias are being conducted.

According to a number of studies, hernias of the MTD can undergo spontaneous resorption in a period of 3 to 36 months. ${ }^{11,12}$ The pain syndrome with medicamental or physiotherapeutic treatment decreased within 3 months. At the same time the overwhelming number of patients experienced the whole period of pain treatment.
Severity of pain at the beginning of the study in accordance with the clinical report of patients and VAS was regarded as pronounced in 12 out of $48(25 \%)$ people, as expressed - in 29 out of $48(60.4 \%)$ and as a rennaya -7 out of $48(14.6 \%)$ patients. ${ }^{13}$

Shockwave therapy has a number of qualities that give grounds for scientific research in the direction of its anti-gambling action. It proved its razvoloknyayuschee and resolving action on the connective tissue and osteophytes, ${ }^{14,6}$ the enhancement of microcirculation and neoangiogenesis, ${ }^{15-17}$ enhancement of cell proliferation and tissue regeneration, ${ }^{18,19,6}$ receipt effects, ${ }^{15,20}$ immunomodulating and immunostimulating effects. ${ }^{18,21,19,22}$ and a number of others. The penetration depth of the shock wave from the standard multi focusing BTL applicator 6000 according to the manufacturer is $50 \mathrm{~mm}$, but the experiments conducted on a dense I gelatin show that at higher powers up to $0.5 \mathrm{mj} / \mathrm{cm} 2$ Depth of passage in a uniform medium is about 60 $\mathrm{mm}$. This in a large number of cases is sufficient to bring the wave directly into the area of the disc defect or adhesive process in the region of the intervertebral foramen. Taking into account the nonlinearity of the displacement of ultrasonic shock waves in complex systems with a large number of phase loci, ${ }^{23,24}$ the presence of secondary and reflected waves ${ }^{23}$ is completely impossible to predict the wave motion in the region of the intervertebral foramen. However, based on the experience of treatment of trigger zones in the lumbar region of the spine, I developed a technology for the treatment of hernias of MPD, called Multifrequency Focusing Shockwave Therapy (MFUVT). The technique is patented. ${ }^{1}$

\section{Materials and methods of research}

In the period from 2012 to 2015. based VR- Clinic study was conducted in patients with hernias MPD lumbar spine in a group of 44 patients, selected according to the routine inspection of the enterprise LLC "Stapvropolstroyoptorg". The size and shape of hernias are determined by the results of MRI studies. Assessed the complaint, the severity of pain on VAS symptom tension ishio-kruralnoy muscle groups (with th Lassega). The patients were divided into 2 groups. 28 patients were the main group; they received specific therapy according to the method of MFVWT. Sixteen patients received nonspecific placebo-treated SWT therapy. The composition of the groups by sex and age is presented in Table 1 . 
At the time of admission, patients from both groups complained of back pain and irradiation of pain to the lower limb. The average pain level at admission of VAS was 7.2 \pm 0.4 . In assessing the patient's condition, the Lassege symptom and stiffness were also taken into account. According to MRI data, all hernias of the lumbar spine of the lumbar spine were revealed in all patients. The size of hernias varies from $5 \mathrm{~mm}$ to $11 \mathrm{~mm}$. In 14 (32\%) patients, a moderate lamination of the posterior longitudinal ligament was observed without a tendency to sequester. In $18(41 \%)$ the tendency to displace the hernia of the MTD in the caudal or axial direction and 11 cases $(25 \%)$ - sequestration in the caudal direction. The summary data of patients are given in (Table 2) (Table 3).

All patients of the main group held courses multifrequency focusing shock wave therapy of 15 treatments, 3-5 bar, for multifrequency scheme in a day to 2000 pulses. Exposure was carried out on the paravertebral area lumbar spine and anatomical projection hernia MPD, through the "sound" of the window with a special laying on the couch with the electric drive. Patients of the second group used therapy with the defocused applicator shock wave therapy, on the waist region, without impacting the anatomical projection of the hernia of the MTD. The course consisted of 10 procedures performed every other day in 2000 pulses, at standard dosages of up to 3 bar, which simulated a placebo-UVT. Along with MIFT for the main group and UHT-placebo for the control group, symptomatic therapy was prescribed to the patients: painkillers, muscle relaxant, sleeping pills. This complex was prescribed for the first group for the first 3-5 days, until the effect of specific therapy was achieved and for the control group for 2-3 weeks. Throughout the course of therapy, patients of both groups were prescribed to wear a corset. Restrictions in labor and mode of activity were not introduced. Patients were trained beforehand in exercise therapy and methods of maintaining proper posture. Perform statistical processing and comparing data in the study and control groups before and after treatment $(p<0,01)$.

Table I Characteristics of patients by sex and age

\begin{tabular}{lllllll}
\hline \multirow{2}{*}{ Group } & \multirow{2}{*}{ Total } & floor & \multicolumn{5}{l}{ age } \\
\cline { 3 - 7 } & $\mathbf{m}$ & $\mathbf{f}$ & Up to $\mathbf{3 0}$ & $\mathbf{3 0 - 5 0}$ & $>\mathbf{5 0}$ \\
\hline Basic & $28(64 \%)$ & $18(64 \%)$ & $10(36 \%)$ & $2(7 \%)$ & $17(61 \%)$ & $9(32 \%)$ \\
Control & $16(63 \%)$ & $10(62.5 \%)$ & $6(37.5 \%)$ & - & $9(56 \%)$ & $7(44 \%)$ \\
& $44(100 \%)$ & $27(61 \%)$ & $17(39 \%)$ & $2(4.5 \%)$ & $34(77.3 \%)$ & $8(18.2 \%)$ \\
\hline
\end{tabular}

Table 2 The main group of patients receiving specific therapy with the Multifrequency Focusing Shockwave Therapy

\begin{tabular}{llllllll}
\hline & Back pain & $\begin{array}{l}\text { Irradiation in } \\
\mathbf{n} \text { / extremity }\end{array}$ & $\begin{array}{l}\text { Assessment of } \\
\text { pain (VASH, } \\
\text { LSH) }\end{array}$ & S-m tension & $\begin{array}{l}\text { Hernia more } \\
\text { than } \mathbf{5} \mathbf{~ m m} \\
\mathbf{( M R I )}\end{array}$ & $\begin{array}{l}\text { The size } \\
\text { of a hernia } \\
\text { on an MRT } \\
(\mathbf{m m})\end{array}$ & $\begin{array}{l}\text { Number of } \\
\text { patients with a } \\
\text { decrease in hernia } \\
\text { by } \mathbf{2} \text { mm or more. }\end{array}$ \\
\hline before & $28(100 \%)$ & $25(89.3 \%)$ & 7.2 & $28(100 \%)$ & $28(100 \%)$ & 6.9 & - \\
after & $\mathrm{I}(3.6 \%)$ & $4(14.3 \%)$ & 1.7 & $1(3.6 \%)$ & $7(25 \%)$ & 4.2 & $21(75.0 \%)$ \\
\hline
\end{tabular}

Table 3 Distribution of patients of the main group by the criterion of a decrease in hernia on MRI

\begin{tabular}{llll}
\hline Total & Significantly $(>\mathbf{2 ~ m m})$ & Doubtful $(<2 \mathbf{~ m m})$ & Without changes \\
\hline 28 & $21(75.0 \%)$ & $4(14.3 \%)$ & $3(10.7 \%)$ \\
\hline
\end{tabular}

\section{Results of the study}

The majority of patients in the main group, $23(81 \%)$, regardless of the severity of the pain syndrome at the beginning of the disease, noted a reduction in pain as early as 2 to 3 procedures (3-5 days after the start of treatment). After that, drug therapy was cancelled. The intensity of pain by the end of the first week on the average on the VAS scale was $3.2 \pm 0.2$. Lack of stiffness was observed in $24(86 \%)$. In $3(11 \%)$ patients with sequestered hernias, the pain was reduced to the 5th procedure (the 9th day after the start of treatment). In 2 $(8 \%)$ than specific manifestations of pain persisted throughout the course of treatment that one of them required the continuous use of pain medication. Two months after the end of the course, an MRI was performed on the same equipment as the primary study. The condition of patients was also assessed. The results of the treatment for the main group are shown in Table 2 and for the control group in Table 4 .
MRT control was performed 2 months after the course of treatment. The average decrease in the size of hernias of MPD was $2.2 \pm 0.7 \mathrm{~mm}$. The distribution of patients of the main group according to the criterion of a decrease in hernia on MRI is presented in Table 3.

Reduction of clinical symptoms and improvement in the wellbeing of patients does not fully correlate with the criterion for reducing hernia in patients of the main group. Clinical improvement was observed more often than a decrease in the size of hernias.

In the control group, the overwhelming number of patients had pain throughout the course, but their intensity decreased to 4-5 points by VAS by 5-7 days from the start of treatment. The reduction in the tension symptom was less pronounced and was observed by 10-14 days. Nevertheless, most patients noted a clinical improvement by the third week of therapy, which gave rise to the withdrawal of drug therapy. The control group also underwent MRI monitoring 2 months after the course of treatment. The results are shown in Table 4. 
Table 4 Control group receiving defocused shock wave therapy by placebo

\begin{tabular}{|c|c|c|c|c|c|c|c|}
\hline & Back pain & $\begin{array}{l}\text { Irradiation in } \\
\mathrm{n} / \text { extremity }\end{array}$ & $\begin{array}{l}\text { Assessment of } \\
\text { pain (VASH, } \\
\text { LSH) }\end{array}$ & S-m tension & $\begin{array}{l}\text { Hernia more } \\
\text { than } 5 \mathrm{~mm} \\
(\mathrm{MRI})\end{array}$ & $\begin{array}{l}\text { The size of a } \\
\text { hernia on an } \\
\text { MRT ( } \mathrm{mm})\end{array}$ & $\begin{array}{l}\text { Number of patients } \\
\text { with a decrease in } \\
\text { hernia by } 2 \mathrm{~mm} \text { or } \\
\text { more. }\end{array}$ \\
\hline after & $8(50 \%)$ & II (68.7\%) & 4.4 & $8(50 \%)$ & 12 (75\%) & 5.8 & No. \\
\hline
\end{tabular}

Dynamic observation for 3, 6, 9 and 12 months showed the preservation of a satisfactory condition in the patients of the main group in the observed period and the absence of clinically significant exacerbations.

\section{Clinical examples}

Sick F, 44 years old, n stumble In February 2015, after months of unsuccessful treatment by a neurologist with complaints of acute pain, radiating to the right $\mathrm{n}$ izhnyuyu limb. He notes periodic weakness in his right leg, a limp. When examining antalgic scoliosis to the left, the relative shortening of the right leg. With th Lassega right 15 degrees, VAS 7-8 points. MRI of predelyaetsya dorsal sided medialparamedian disc herniation L3/L4, with a tendency to sequestration of up to $0.8 \mathrm{~cm}$, extending to the right intervertebral foramen, with its contraction, having caudal spread sequestering component along the body of L4 vertebra to $0.5 \mathrm{~cm}$ in the right lateral pocket, with compression of the right nerve root, compressing adjacent sections of the dural sac (Figures 1A \& 1B).

In February-March of 2015 MFUVT course of 15 treatments was carried out. The conduct and course of UHT MF, pain decreased during the first 5 days, with th Lassega - 80 degrees, no lameness, pain on the VAS scale of 4 points. MRI (Figures 2A \& 2B) Performed 2 months after the end of the UHT MF: Dorsal sided paramedian hernia $\mathrm{L} 3 / \mathrm{L} 4$ disc, up to $0.5 \mathrm{~cm}$, extending into the intervertebral foramen, on both sides, with their restrictions, as well as several caudal into the right lateral pocket, compressing the dural sac. Sequestration is not noted.

Patient C, 31 years old. Has acted with complaints on sharp pains in a loin, pulling pains in the left leg, numbness of the left leg, paresthesia. When examining antalgic scoliosis to the left, weakness in the right $\mathrm{n} /$ extremity, it's shortening by $1.5 \mathrm{~cm}$, with Lassega $\mathrm{s}$ sharply positive on the right. MRI (ris.5-6) Disc L 4-5: moderate decrease in disc height and intensity MR - signal. Op. posterior median disc hernia with left lateralization of $17 * 7.9 * 7.9 \mathrm{~mm}$, with a tendency to sequestration (Figures 3A \& 3B).

In the period from May to June 2015, patient S. was given a course of MFVVT from 15 procedures. During the first week, the patient's condition stabilized, pain and irradiation in the limb decreased, antalgic scoliosis is not detected, a symptom of tension of 50 degrees. By the end of the course of treatment, the patient's condition is good, there is no pain in the lower back. Preserve the area of numbness in the left foot. Inspection after 2 months did not reveal pathological abnormalities. MRI from 27.08.15. (Figures 4A \& 4B). Drive L 4-5: moderate decrease in disc height and intensity MR - signal. Op. posterior protrusion of the disc up to $4 \mathrm{~mm}$. Bone swelling of adjacent vertebrae.
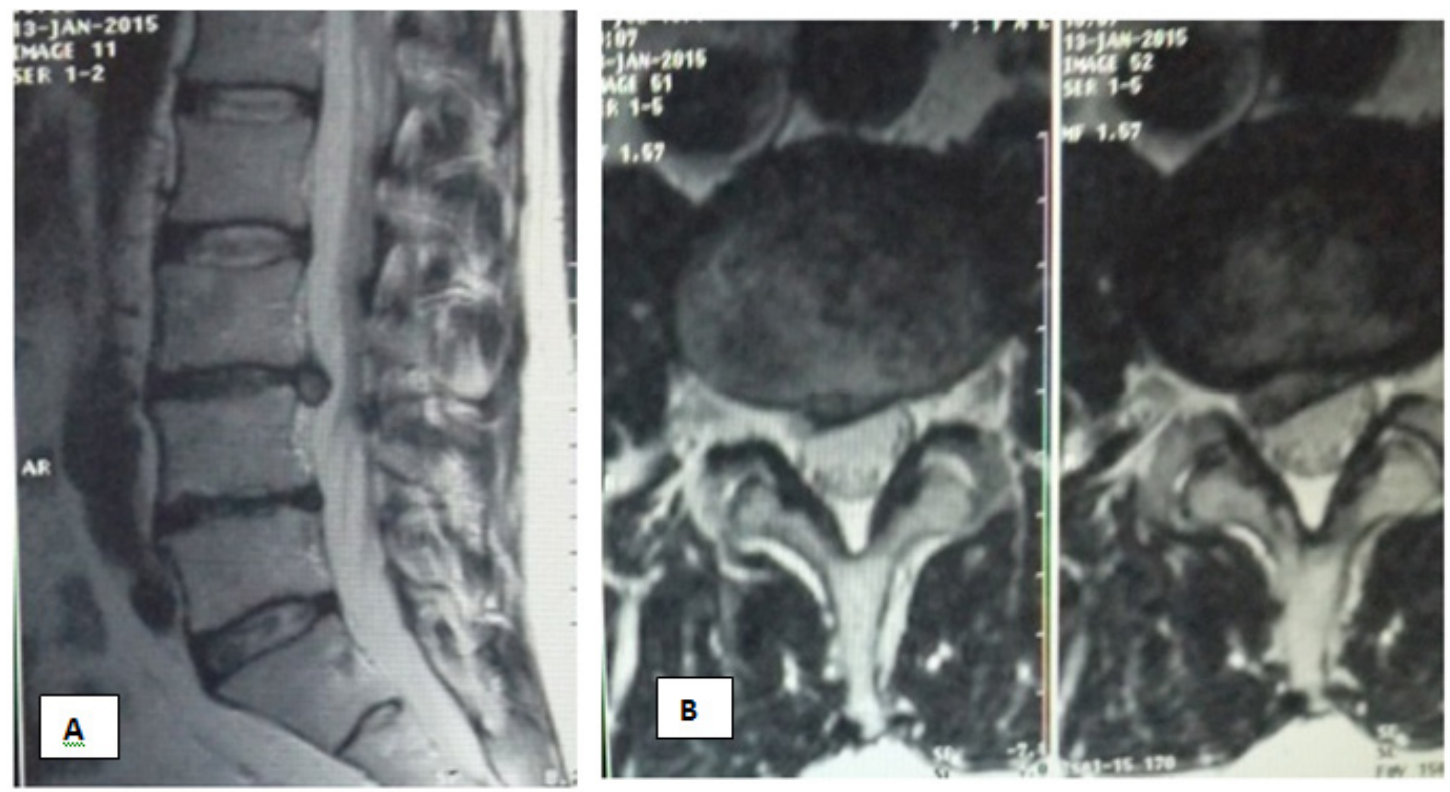

Figure I Patient F. MRI examination before treatment. 

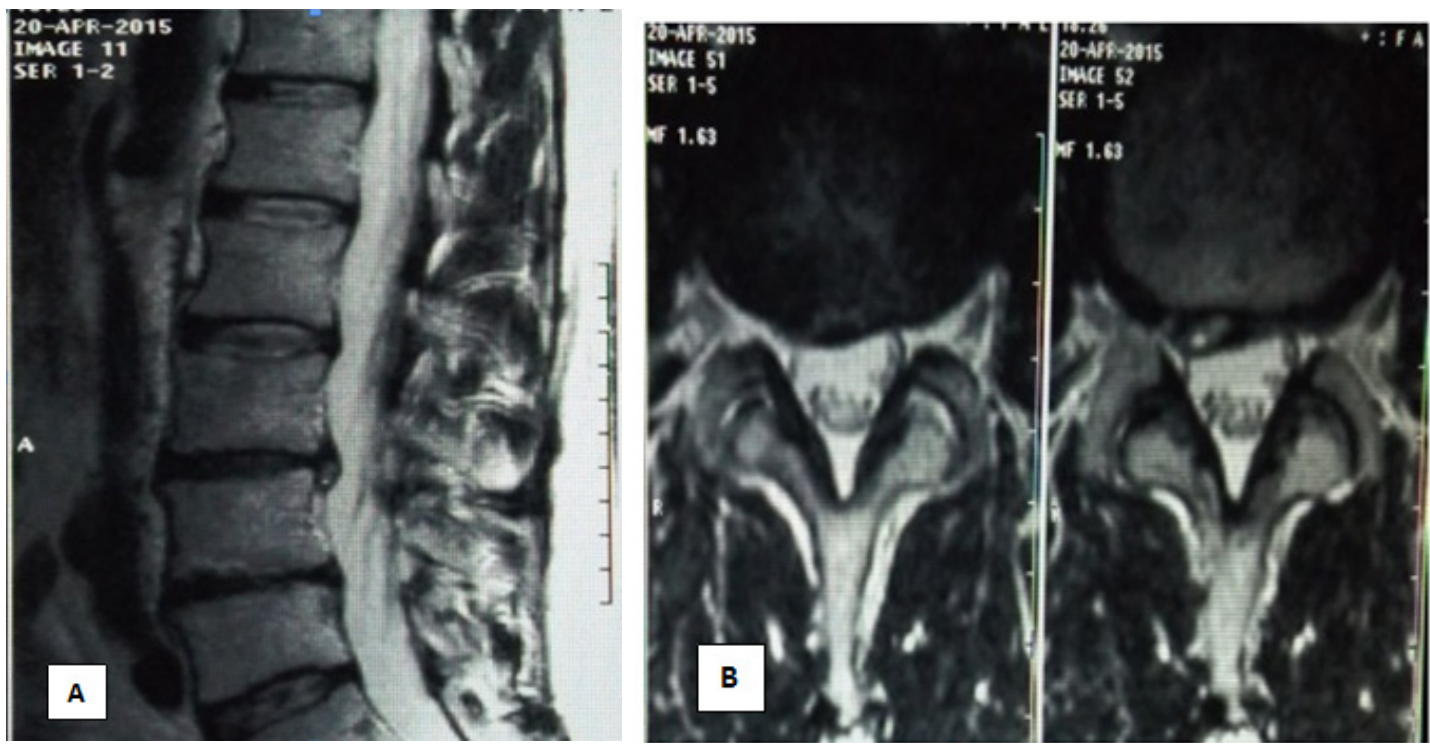

Figure 2 Sick F. Decrease in the size of herniated MTD after the course of UHT.
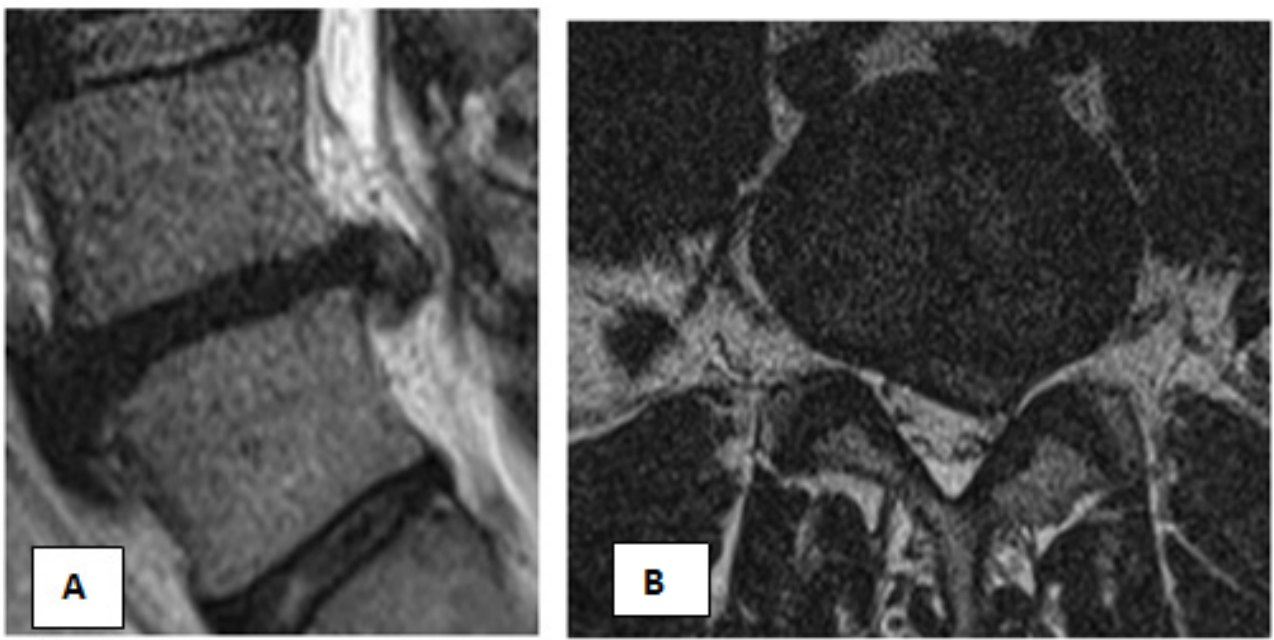

Figure 3 Patient $\mathrm{C}$, before treatment.
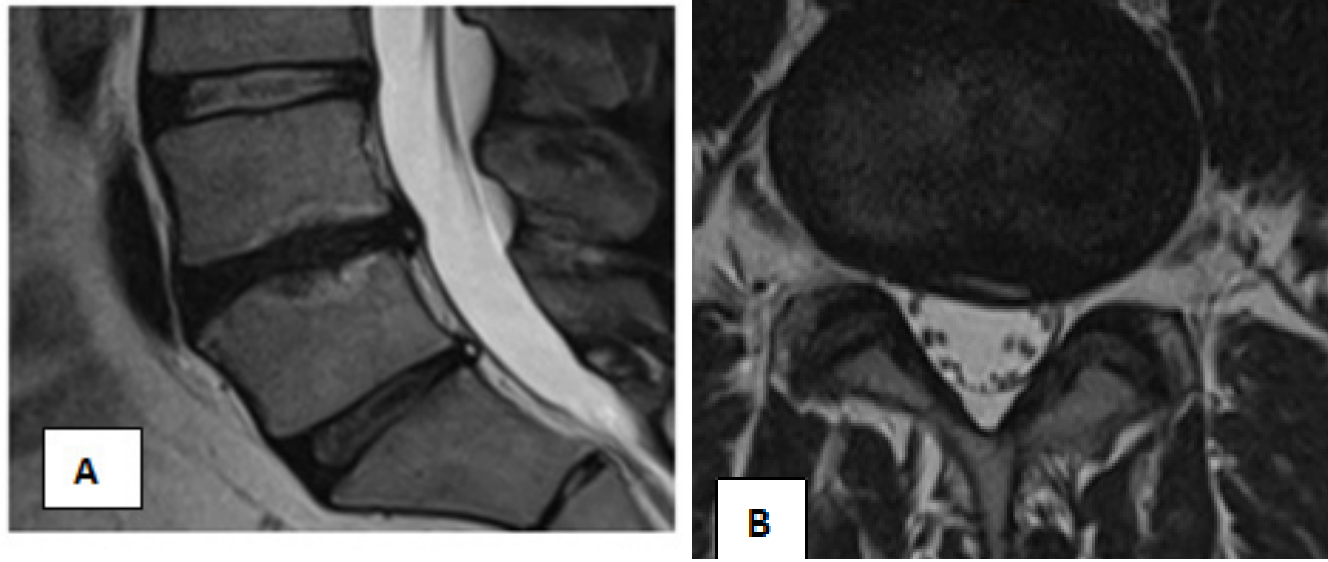

Figure 4 Patient S.After the course of MFUVT.

Citation: Mikhailovich OL. Opportunities multifrequency focusing shock wave therapy in lecheni and intervertebral hernias of the lumbar spine. Int Phys Med Rehab J. 2018;3(5):448-452. DOI: I0.15406/ipmri.20I8.03.00I44 


\section{Discussion}

Shock wave therapy because of its unique biophysically $\mathrm{x}$ the properties in definitely has a positive effect on all kinds of back pain, including pain caused by degenerative-dystrophic diseases and hernial entities MTD. However, the non-specific effect of UHT does not lead to acceleration in the reduction of hernial protrusion.

The factors that contribute to the reduction of the hernia are presumably the following:

a. Cavitational evaporation of a hernial fluid,

b. Reduction of disc edema in the course of neoangiogenesis.

c. Active immunomodulating and immunopotentiating effect of the shock wave, realized primarily through the mechanism of proliferation of neutrophils and fibroblasts/fibroblasts in the area of the hernial process. Resorption hernia apparently was active fibroklastov and its subsequent regeneration with the formation of connective tissue scar proliferating fibroblasts.

Not unimportant factor in the rapid clinical improvement is the impact shock wave at the connective tissue adhesions and osteophytes, as well as a positive effect on the course spondylarthrosis, spondylosis and spondliloliza. Nevertheless, the use of UHT hernia requires careful process carried purity techniques, knowledge of anatomical features of the patient, its correct placement and correct use of different pulse sequences, a large number of procedures. This approach is implemented in the technology of MIFWT, which has shown its effectiveness.

\section{Conclusion}

Thus, the use of multifrequency focusing shock wave therapy (MIFT) is preferred when using UHT in patients for lumbar pain. It leads to rapid reduction of pain syndrome and neural manifestations of radicular compression, and also accelerates the decrease in the size of the hernia, which is much less observed in the case of non-specific UHT.

\section{Acknowledgments}

None.

\section{Conflict of interest}

The authors declare no conflict of interest.

\section{References}

1. Ovcharenko LM. The method of treatment of hernia of the intervertebra discs. Patent for invention № 2577239; 2014.

2. Gerasimov MV. Minimally invasive discectomy in the surgical treatment of lumbar osteochondrosis/ MV Gerasimov // Materials of the 3rd AllRussian Congress of Neurosurgeons.-SPb; 2002;239-240.

3. Filatov VS. Compressing factors and indications for surgical treatment in patients with persistent compression lumbar and sacral radiculopathy with degenerative spine injuries ABSTRACT theme of VAK.

4. Anthony H, Wheeler MD, editors. Stephen A, Berman. Translation review low back pain and sciatica, published in Medscape; 2013.

5. Jensen MC, Brant ZMN, Obuchowski N, et al. Magnetic resonance imaging of the lumbar spine in people without back pain. Engl J Med N. 1994;331(2):69-73

6. Powell MC, Wilson M, Szypryt P, et al. Prevalence of lumbar disc degeneration observed by magnetic resonance in symptomless women The Lancet. 1986;2(8520):1366-1367.

7. Weinreb JC, Wolbarsht LB, Cohen JM, et al. Prevalence of lumbosacral intervertebral disk abnormalities on MR images in pregnant and asymptomatic nonpregnant women. Radiology. 1989;170(1 Pt 1):125128.

8. Wiesel SW, Tsourmas N, Feffer HL, et al. A study of computer-assisted tomography. I. The incidence of positive CAT scans in an asymptomatic group of patients. Spine.1984;9(6):549-551.

9. Haldeman S. North American Spine Society: failure of the pathology model to predict back pain. Spine. 1990;15(7):718-724.

10. Aul S. Video endoscopy in the surgical treatment of lumbar intervertebral hernia Thesis abstract on HAC 14.00.28.

11. Kurilina LR, Velichko MI, Sokolova EV, et al. Spontaneous resorption of herniated intervertebral discs of the lumbar spine. $M$ pocket medical almanac. 2013;198-201.

12. Eiichi T, Masaya T, Kimio S, Natural history of lumbar disc hernia with radicular leg pain: Spontaneous MRI changes of the herniated mass and correlation with clinical outcome. J Orthop Surg. 2001;9(1):1-7

13. Oleinikov LL, Remnev LG. Conservative treatment of hernias of lumbar intervertebral disc. International Journal of Applied and Basic Research. 2011;(12):69.

14. Braun W, Claes A, Ruter A, et al. Effects of extracorporeal shock waves on the stability of the interface between bone and polymethylmethacrylate: An in vitro study on human femoral segments. Clin Biomech. 1992;7:4754 .

15. Brendel W, Delius M, Goetz A. Effect of shock waves on the microvasculature. Prog Appl Microcirculation. 1987;12:41-50.

16. Brummer F, Brauner T, Hulser D. Biological effects of shock waves. World J Urol. 1990;8:224-232.

17. Holfeld J, Tepeköylü C, Blunder S, et al. Low energy shock wave therapy induces angiogenesis in acute hind-limb ischemia via VEGF receptor 2 phosphorylation. PLoS One. 2014;9(8):e103982.

18. Zhiyu C, Frank F, Oleh A, et al. Effects of Shock waves on expression of IL-6, IL-8, MCP-1, and TNF- $\alpha$ expression by human periodontal ligament fibroblasts: of an with in vitro study. Med Sci Monit. 2016;22:914-921.

19. Weihs AM, Fuchs C, Teuschl AH, et al. Shock wave treatment enhances cell proliferation and improves wound healing release-atp by coupled extracellular signal-regulated kinase (ERK) Activation. J Biol Chem. 2014;289(39):27090-27104.

20. Nikolaus BMC, Nicholas BA, Stefan M, et al. Radial shock wave devices generate cavitation. PLoS One. 2015;10(10): e0140541.

21. Agostino MC, Craig K, Tibalt E, et al. The Shock wave as with the Biological therapeutic tool: the from mechanical stimulation to the healing and recovery, through mechanotransduction. Int J Surg. 2015;24(Pt B):147-153.

22. Wang CJ. An overview of shock wave therapy in musculoskeletal disorders. Chang Gung Med J. 2003;26(4):220-232.

23. Karzova MM. Non-linear effects in the reflection and focusing of discontinuous acoustic waves in atmospheric and medical acoustics problems Specialty: 01.04.06 - acoustics, Dissertation for a scientific degree candidate of physical and mathematical sciences.

24. PB Rosnitsky, PV Yuldashev, VA Khokhlova. Moscow State University. MV. Lomonosov Moscow State University, Faculty of Physics Influence of the angular aperture of medical ultrasonic radiators on the parameters of a nonlinear shock wave field in focus. Acoustical journal, 2015;61(3):325-332. 